\title{
Reasons why patients with primary health care problems access a secondary hospital emergency centre
}

\author{
Juanita Becker, Angela Dell, Louis Jenkins, Rauf Sayed
}

Background. Many patients present to an emergency centre (EC) with problems that could be managed at primary healthcare (PHC) level. This has been noted at George Provincial Hospital in the Western Cape province of South Africa.

Aim. In order to improve service delivery, we aimed to determine the patient-specific reasons for accessing the hospital EC with PHC problems.

Methods. A descriptive study using a validated questionnaire to determine reasons for accessing the EC was conducted among 277 patients who were triaged as green (routine care), using the South African Triage Score. The duration of the complaint, referral source and appropriateness of referral were recorded.
Results. Of the cases $88.2 \%$ were self-referred and $30.2 \%$ had complaints persisting for more than a month. Only $4.7 \%$ of self-referred green cases were appropriate for the EC. The three most common reasons for attending the $\mathrm{EC}$ were that the clinic medicine was not helping $(27.5 \%)$, a perception that the treatment at the hospital is superior (23.7\%), and that there was no PHC service after-hours (22\%). Conclusions. Increased acceptability of the PHC services is needed. The current triage system must be adapted to allow channelling of PHC patients to the appropriate level of care. Strict referral guidelines are needed.

S Afr Med J 2012;102(10):800-801. DOI:10.7196/SAMJ.6059
Emergency centres (ECs) provide emergency care to people with acute trauma and illness who require the services and expertise available at a hospital. ${ }^{1}$ However, the presenting complaints at an EC overlap considerably with those encountered at primary healthcare (PHC) level. ${ }^{2}$ Studies suggest that one-third to two-thirds of patients attend ECs with problems that could have been managed at a PHC level. ${ }^{2-5}$

The South African Triage Score (SATS) (previously known as the Cape Triage Score $)^{6-7}$ is routinely used at George Provincial Hospital to triage patients presenting to the EC to determine their acuity level and prioritise them accordingly. The five categories are red (immediate care), orange (very urgent care), yellow (urgent care), green (routine care) and blue (dead).

A retrospective descriptive study at George Hospital in May 2010 to determine the after-hours case mix and workload ${ }^{8}$ demonstrated that $65 \%$ of patients who presented to the EC after-hours were triaged green. This demonstrated that many low-acuity patients are seen in the EC. From the perspective of service delivery this 'inappropriate' attendance is problematic, as it competes for the attention of EC staff and potentially compromises the quality of care for more serious cases needing urgent treatment. A need was identified to determine the patient-specific reasons for presenting to a secondary hospital EC with PHC problems.

George Hospital, George, Western Cape

Juanita Becker, MB ChB, first-year intern

Angela Dell, BSc, MB ChB, community service medical officer

Department of Family Medicine, George Hospital, George, Western Cape, and Stellenbosch University, Tygerberg, Western Cape

Louis Jenkins, MFamMed, FCFP (SA)

School of Public Health and Family Medicine, Faculty of Health Sciences, University of Cape Town Rauf Sayed, MSc

Corresponding author: A Dell (angelajdell@gmail.com)

\section{Methods}

George Hospital is a secondary (level 2) provincial hospital in the Western Cape province of South Africa, providing healthcare to the population of the Eden and Central Karoo districts. The population numbers about 512 000, of whom about 140000 live in George. ${ }^{9}$ The health needs of the George community are served by 10 PHC clinics, a few mobile clinics, private practitioners, the private Mediclinic and George Hospital. There is no district hospital in the George subdistrict, and the PHC clinics offer no after-hours services. Everyone requiring healthcare after-hours, over weekends and on public holidays must therefore access the EC at George Hospital or use the private sector.

The study was conducted between 5 March and 5 April 2012. A validated questionnaire ${ }^{10}$ was refined to our setting after conducting a pilot study which included 30 patients.

Convenience sampling was used. The SATS was used to identify all patients triaged as green. A questionnaire was then placed in the patient's folder by the nurse practitioner who did the triaging. The doctor who subsequently saw the patient obtained informed written consent from the patient. The duration of the presenting complaint, the referral source and whether the referral was appropriate were obtained. An inappropriate referral was considered to be a patient who was not admitted, did not need a procedure or special investigation, or was not referred to a specialty. The patient was then asked to select his or her two or three main reasons for attending the EC from a list of 17 options.

Data were captured and analysed using Microsoft Excel 2003 software. Data were mainly descriptive in nature. Statistical support was offered by the School of Public Health and Family Medicine, University of Cape Town.

The study was approved by the Human Ethics Committee of the University of Cape Town.

\section{Results}

A total of 277 patients agreed to participate, giving a response rate of $29.4 \%$. Forty-two questionnaires could not be used because data were incomplete. Of the remaining 235 patients, 44\% were male and 56\% female with a mean age of 31.5 years.

Of patients presenting with problems, $37 \%$ had had the complaint for 24 hours to 1 week, and $30.2 \%$ for more than 1 month. 
Of patients $88.9 \%$ were self-referred; of these referrals $4.8 \%$ were considered appropriate. Of the $10.3 \%$ referrals from general practitioners and clinics, $16 \%$ were appropriate for the EC.

The common self-reported reasons for attending the EC were: $(i)$ the clinic medicine was not helping $(27.5 \%)$; (ii) a perception that the treatment at the hospital was superior to that at the clinic (23.7\%); (iii) lack of a PHC service after-hours in the George sub-district (22\%); (iv) too-long clinic waiting times (14\%); $(v)$ patients being referred to the EC (12.3\%); and ( $v i)$ that patients could have 'special tests' at the hospital (11.9\%).

\section{Discussion}

Of the patients $88.9 \%$ were self-referred, and only $4.8 \%$ of these were appropriate for the EC. This finding correlates with a study at Paarl Hospital, a secondary (level 2) hospital in the Western Cape, where $88.2 \%$ of patients were self-referred. ${ }^{11}$ At New Somerset Hospital in Cape Town (an urban setting) there was a self-referral rate of $41 \%{ }^{12}$ This could indicate that a more efficient and controlled referral system, and/or more extended PHC services, exist in South Africa's larger cities compared with rural towns such as George and Paarl. Although the appropriateness of referrals of patients from PHC doctors and nurses was better, the figure of $16 \%$ is still very low, indicating that PHC doctors and nurses still need information and support on how to refer patients effectively and efficiently.

Of patients who presented with problems, $30.2 \%$ had had the problem for more than 1 month. Patients with chronic complaints tend to use several health services, including the level 2 hospital EC, hoping for a cure, which is often not forthcoming. This situation results in frustrated patients, who are not necessarily cured in the EC, and frustrated health staff, who are distracted from acute emergencies by chronic complaints.

Access to emergency care is a constitutional right. ${ }^{13}$ It is evident from the reasons given by patients that there is a sense of entitlement to access hospital care for any complaints. For various reasons, it seems that many patients repeatedly access the EC with problems that should be addressed in PHC. Factors of access, convenience, health beliefs and previous experience with the health service must be considered. Dr Aaron Motsoaledi, Minister of Health, voiced his concern that going to the hospital instead of the clinic has become the norm in South Africa, and that this trend is crippling our country's health system. 'People in South Africa are not utilising clinics. They are utilising tertiary hospitals. This is not how health care must be conducted. You don't wake up and go to the highest hospital. You start at clinics. ${ }^{14}$

One patient said that her reason for coming to the EC and not the clinic was: 'They only see a set number of patients at the clinic each day; I know that I can come to the hospital at any time and they will not chase me away if I don't have a letter or an appointment.' This indicates a belief among patients that the EC will attend to their problem, even if it is not an emergency. This was emphasised by another patient: 'I just don't believe in clinics, their treatment never works.' Some patients are prepared to wait for many hours to be seen in the EC, instead of going to their nearest PHC clinic, as they seem to prefer hospital treatment, reinforcing the second most common reason given for attending the EC. Some patients also reported that they are not examined at the clinic and are only prescribed medication based on their complaints. This may explain some of their health-seeking behaviour.

Several patients presented with direct requests, such as 'I want a chest X-ray' or 'I want a head scan', in keeping with the $11.9 \%$ who reportedly accessed the EC to have special tests done. Dale et al. ${ }^{2}$ showed that many patients who present with primary healthcare problems do not need an investigation, minor surgical intervention or referral. However, many patients in this category receive interventions likely to support their decision to attend the EC rather than PHC clinics. This may reflect a clinical approach of junior medical staff, including a propensity to intervene, and indeed at a secondary level hospital much of the workforce in the EC may comprise junior medical officers, community service doctors and interns.

The Western Cape Emergency Case Load Management Policy (ECLMP) was developed to improve the efficiency of ECs in the Western Cape (K C Househam, Western Cape Emergency Case Load Management Policy (ECCLMP), draft 2.4, April 2012 - unpublished data). It proposes that patients that have been triaged by a senior healthcare professional and who do not require further emergency treatment should be referred to the appropriate facility or service.

\section{Limitations}

This is an observational study with some degree of observation bias introduced in the calculation of the triage score. However, patients are generally triaged by experienced nurse practitioners and the triage score can be trusted to be accurate in the majority of cases. Bias may have been introduced by having the EC doctors assist patients to complete the questionnaire. Fixed question ordering bias may have played a role and was not excluded.

\section{Conclusions and recommendations}

There is a need to increase the acceptability of PHC clinic services to the public. Focused public education campaigns on the PHC services available and appropriate use of the emergency services are necessary. A 24-hour PHC facility would reduce the number of green patients seen after-hours in the EC in George. Alternatively, the appointment of clinical nurse practitioners in hospitals would provide after-hours $\mathrm{PHC}$ services without interfering with the EC's primary function. The triage system must be adapted to allow channelling of PHC patients to the appropriate level of care. It would be helpful to create a standard letter that can be used when referring a patient to the appropriate level of care.

Acknowledgements. We give special thanks to the EC doctors and nurses who helped with administration of the questionnaires, and the hospital management who supported the study.

\section{References \\ 1. Murphy AW. 'Inappropriate' attenders at accident and emergency departments I: definition, incidence and reasons for attendance. Fam Pract 1998;15:23-32. [http://dx.doi.org/10.1093/fampra/15.1.23] 2. Dale J, Green J, Reid F, Glucksman E Primary care in the accident and emergency department: Prospective identification of patients. BMJ 1995;311:423-426. [http://dx.doi.org/10.1136/bmj.311.7002.423] identification of patients. BM/ $1995 ; 311: 423$-426. [htp://dx.doi.org/ $/ 0.1136 / \mathrm{bmj}$.311.7002.423] Liggins K. Inappropriate attendance at accident and emergency departments: a liter
Nurs 1993:18(7):1141-1145. [http://dx.doi.org/10.1046/j.1365-2648.1993.18071141.x] Wise M. Inappropriate attendance in acciden dx.doi.org/10.1016/50965-2302(97)90091-6 \\ 5. Gill JM. Nonurgent use of the emergency department: appropriate or not? Ann Emerg Med 1994;24:953 957. [http://dx.doi.org/10.1016/S0196-0644(94)70213-6] \\ 6. Gottschalk S, Wood D, Devries S, et al. A triage system for the Cape Town area: proposal from the Cape Triage Group. Emerg Med J 2006;23:149-153. [http://dx.doi.org/10.1136/emj.2005.028332] \\ 7. Gottschalk SB, Wood D, De Vries S, Wallis LA, Bruijns S. The Cape Triage Score: a new triage syster for South Africa. Proposal from the Cape Triage Group. Emerg Med J 2006;23:149-153. [http://dx.do org/10.1136/emj.2005.028332] \\ 8. Van Wyk PS. After hours case mix at George Provincial Hospital Emergency Centre: a descriptive stud Unpublished MMed thesis, Stellenbosch University, 2010 \\ 9. Statistics South Africa. Census 2001: Primary Tables Western Cape - 1996 and 2001 Compared. Pretoria Statistics South Africa, 2004. http://www.statssa.gov.za/census01/html/c2001 primtables.asp (accessed April 2012). \\ 10. Masso M, Bezzina AJ, Siminski P, Middleton R, Eagar K. Why patients attend emergency departments for conditions potentially appropriate for primary care: Reasons given by patients and clinicians differ Emerg Med Australas 2007;19(4):333-340. [http://dx.doi.org/10.1111/j.1742-6723.2007.00968.x] \\ 11. Hanewinckel R, Jongman P, Wallis LA, Mulligan MT. Emergency medicine in Paarl, South Africa: cross-sectional descriptive study. Int J Emerg Med 2010;3(3):143-150. [http://dx.doi.org/10.1007/s12245- 010-0185-9] \\ 12. Hodkinson PW, Wallis LA. Cross-sectional survey of patients presenting to a South African emergency centre. Emerg Med J 2009;26(9):635-640. [http://dx.doi.org/10.1136/emj.2008.063362] \\ 13. South African National Health Care Act. No 61 of 2003. Government Gazette 2004; vol. 469. Cape Town, 23 July 2004. www.info.gov.za (accessed 1 February 2012) \\ 14. Motsoaledi A. Health system needs overhaul. News 24, 26 anuary 2011. www.news24.com/SouthAfrica/} Politics/Motsoaledi-Health-system-needs-overhaul-20110126 (accessed 1 February 2012) 